\title{
A BENIGN TYPE OF RHEUMATIC FEVER
}

BY

\section{W. L. ACKERMAN}

A diagnosis of rheumatic fever conjures in the minds of most physicians a serious disease demanding prolonged rest in bed, suggesting the likelihood of cardiac damage, and producing subsequent invalidism to a varying degree. Copeman (1944) described 42 cases of febrile polyarthritis of acute onset which he states were " non-epidemic, of short duration, and probably not uncommon." None of his cases developed cardiac damage, and all occurred in patients whose previous resistance to disease appears to have been exceptionally high. For this benign syndrome he suggests the name "acute febrile myalgia" as being better than "benign rheumatic fever" for several reasons, in spite of the prominence of the arthritic manifestations.

Ferguson (1944), in reviewing 243 such cases occurring in the Canadian Army, suggested the appellation "acute febrile polyarthritis" for a condition in adults simulating rheumatic fever, but which has not the gloomy prognosis of this disease as seen in children. The diagnosis of rheumatic disease was usually followed in the Canadian Army by discharge from the Service (162 out of 243). In his opinion at least half of those who developed the condition and were discharged could continue in military service. W. Tegner (1944), the reviewer of Ferguson's article, could not agree that a change in nomenclature is needed. He, however, agreed that a diagnosis of rheumatic fever should not automatically entail discharge from the Army.

The 47 Service patients discussed in this paper suffered with this " benign type" of rheumatic fever and have been seen within the last $2 \frac{1}{2}$ years. They showed an acute febrile illness with non-suppurative polyarthritis simulating classical rheumatic fever, yet with features which differentiated them from this serious disease, and enabled a shorter period away from duty to be imposed, and a more optimistic prognosis to be given, which within the limits of a relatively short follow-up proved to be justified. During this period only 7 cases of classical rheumatic fever with endocarditis were seen, and in none of these was the attack the primary one.

\section{Typical Case Reports}

\section{CASE 1}

Aged 28. No previous history of rheumatic fever or rheumatism. No history of tonsillitis-tonsils removed 10 years ago. For past four months had been exposed to severe cold and wet and had not been able to dry his clothes properly. Two days before admission began to complain of pain and stiffness in several joints, particularly L. elbow and R. knee. The following day both knees became obviously swollen.

On Admission-May 7 : O.E. Well developed man. T. $102 \cdot 8^{\circ}$, P. 102 . Does not look ill. Slight sweating. No tonsil remnants seen. Marked effusion into both knee joints; periarticular swelling L. ankle. These 3 joints are painful on movement and palpation. Less severe pain $R$. wrist and $R$. shoulder. Treated with sod. sal. grs. 120 daily.

Progress.-May 9: T. 98.6, P. 74. Pain less, has almost disappeared from $\mathbf{L}$. ankle, R. wrist, and $\mathbf{R}$. shoulder. Knees still painful and swollen. B.S.R. $=42 \mathrm{~mm}$. in 1 hour. May 11: T. $98 \cdot 2^{\circ}$, P. 70. Disability limited to both knees which are still moderately swollen and painful. May 13: T. normal, P. 70. No swelling of knees. B.S.R. $=42$. May 14: Sod. sal. reduced to 90 grains daily. B.S.R. $=40$. Slight stiffness in knees. R.B.C. $=5,200,000$. C.I. $=1 \cdot 0$. May 20: Feels well; remains apyrexial; slight stiffness in knees. May 24: B.S.R. $=24$. Sod. sal. stopped. May 28: B.S.R. $=14$. Begins getting up for gradually increasing periods. Heart remains unaffected. June 16: Up all day. No complaints. B.S.R.=10. June 28: Returned to duty. Well.

CASE 2

Aged 26. For past two weeks had been undergoing severe training during which he had frequently become soaked by rain: " had been living rough." Frequent twinges of pain in limbs and back during this time. For past three days had had difficulty in performing his duties owing to pain in ankles and feet. Several mild attacks of lumbago during his training as a soldier. No history of sore throat or previous rheumatism.

Dec. 12: O.E. Well developed man. T. $102^{\circ}$, P. 96. Complains of pain in various joints. Joints affected are: both ankles (some periarticular swelling with pain on movement), both knees (pains and stiffness only), and L. shoulder (restricted movement owing to pain). Given sod. sal. grs. 120 daily. Dec. 13: T. 102.6 P. 100. Pain more severe. Effusion into L. knee joint. B.S.R. = 34. Dec. 14: T. $102^{\circ}$, P. 96. No change in physical signs. Sod. sal. increased to 150 grs. daily. Dec. 15: T. normal, P. 80. Periarticular swelling of ankles had disappeared, some pain still present on movement. Effusion still persists in L. knee. Dec. 18: Disability limited to very slight effusion L. knee. B.S.R. $=30 \mathrm{~mm}$. R.B.C $=4,800,000$. C.I. $=1 \cdot 2$. Sod. sal. reduced to 90 grs. daily. Dec. 24: No symptoms. B.S.R. $=24 \mathrm{~mm}$. Sod. sal. stopped. Dec. 16: B.S.R. $=16 \mathrm{~mm}$. Jan. 1: B.S.R. $=12$. Heart has remained unaffected throughout. Jan 24: Uneventful progress. Discharged to duty. 
CASE 3

Aged 23. 2 days sore throat. P.H. Thinks he had rheumatic fever aged 10 . Nil since. In bed 5 weeks. Has been susceptible to sore throats with occasionally a muscular pain in his back; otherwise has always considered himself to be very fit.

Sept. 12: O.E. T. $103 \cdot 4^{\circ}$, P. 100 . Bilateral acute follicular tonsillitis with peritonsillar induration. Swab showed hæmolytic streptococci. No other abnormal physical signs on examination. Sulphonilamide 2 grammes stat. and 1 gramme 4-hourly. Sept. 14: T. normal. Throat has responded well to sulphonilamide. Peritonsillar induration has disappeared. Slight follicular tonsillitis. Sept. 17: T. 102, P. 94. Complains of pain in knees and wrists. Effusion into L. knee. No (obvious tonsillitis. Sulphonamide stopped. (36 grammes) Sod. sal. grs. 150 daily begun. Sept. 18: T. 102.6 P. 100. Pain more severe. Effusion L. knee has increased. Periarticular swelling and puffiness $\mathrm{L}$. ankle. c/o severe pains in wrist; $L$. wrist slightly swollen. B.S.R. $=48 \mathrm{~mm}$. Sept. 20: T. 99.2 ${ }^{\circ}$, P. 96. Effusion both knees, less pain. Induration around $L$. ankle has disappeared. Heart $=$ N.A.D. R.B.C. $=5,000,000$. C.I $=1 \cdot 1$. Swab from tonsillar region $=$ haemolytic streptococci. Sept. 22: T. normal, P. 88. Effusion much less in knees. Slight pains in other joints. Sept. 26: T. normal, no effusions. Very slight pain in knees. Sod. sal. reduced to 90 grs. daily. Sept. 29: B.S.R. $=20$. No symptoms or signs. Sod. sal. ceased. Oct. 4: B.S.R. $=16 \mathrm{~mm}$. Oct. 10: B.S.R. =4. Begins to get up. Oct. 21: Discharged to light duty. Oct. 24: Returns for tonsillectomy: has had no recurrence of symptoms. Heart-N.A.D.

\section{CASE 4}

Aged 32. Aug. 29: Pain and swelling in both ankles -he thought it due to route marches. Persisted for two days but not sufficiently severe to prevent his embarking in a landing craft. No previous rheumatic or other illness. No undue exposure to cold or damp. Tonsils removed as a child. Sept. 3: Recurrence of pain in ankles, also appeared in knees, persisted. Sept. 5: O.E. T. $102 \cdot 8^{\circ}$, P. 98 . Marked painful effusions into knees, L. and R. Both ankles swollen and painful. Tenderness and induration L. wrist. Pain on moving L. elbow and shoulder. Tonsils had been radically removed. Sod. sal. grs. 150 daily. Sept. 7: T. $102^{\circ}$, P. 90. Knees as before, both wrists now affected. Ankles appear normal except for slight pain on movement. B.S.R.=32 mm. Sept. 9: T. normal, P. 74. Slight effusion both knees. Wrists less swollen. Sept. 12: Remains apyrexial. P. 68. Subjective symptoms in knees. Cease sod. sal. Sept. 18: Feels well. Heart remains clinically unaffected. B.S.R. $=32 \mathrm{~mm}$. Sept. 28: B.S.R. $=20$. Allowed up for increasing periods. Oct. 3: Sudden recurrence of pain in knees, wrists and L. ankle. Oct. 3: T. $101^{\circ}$, P. 80 . Mild effusion into knees. B.S.R. $=28 \mathrm{~mm}$. Sod. sal. grs. 150 recommenced. Oct. 5: T. normal, P. 76. Pain in joints much less. Slight effusion still present in knees. Oct. 9: Remains apyrexial. P. 68. No pain. No effusion. Sod. sal. reduced to grs. 90 daily. Oct. 14: Sod. sal. ceased. Oct. 18: B.S.R. $=20 \mathrm{~mm}$. Oct. 26: Feels well. B.S.R. $=8 \mathrm{~mm}$. Recommenced getting up. Nov. 16: Discharged. Heart remains unaffected throughout illness.

SYMPTOMS AND SIGNS

The average age of these 47 male patients was 25.2 years. The onset of the illness was fairly sudden with pyrexia (up to $103 \cdot 8^{\circ}$ ). Stiffness and pain occurred in the joints, rapidly followed in many by effusions. The joints involved were chiefly the ankles, knees, wrists and elbows, the hips and shoulders being occasionally implicated. Moderate periarticular swelling was frequently present in the wrists and ankles. There was very little tendency for the pains to flit from joint to joint. There was generally no previous history of rheumatism or other serious illness. The patient rarely felt acutely ill, the discomfort associated with the joints being the outstanding complaint. Profuse sweating was not noticed. Any increase in the pulse rate corresponded with the pyrexia. No rheumatic nodule nor any of the erythemata was seen. In all the patients (except 2), the blood sedimentation rate was raised, generally $30-40 \mathrm{~mm}$. in one hour (Wintrobe). Examination of the red blood cells and haemoglobin in 19 cases showed a normal count.

\section{PROGRESS}

All were treated with sodium salicylate 120 150 grs. daily. The clinical improvement was rapid. The swelling of the joints usually disappeared in 5 days although arthritic discomfort was frequently perpetuated for several more days. Almost invariably the patient quickly protested at being confined to bed. All but 2 were kept in bed until the B.S.R. had fallen to normal (average 24 days) after which they were allowed up for gradually increasing periods, as the sedimentation rate is considered by the majority of investigators as the most important simple laboratory test of rheumatic activity. Recurrences and relapses, so common in rheumatic fever, were rare. Three patients relapsed soon after beginning to get up. Two of these had been allowed to exercise before the B.S.R. had fallen to satisfactory levels. The response of these relapses to bed and salicylates was as prompt as in the original attack. None developed endocarditis or myocarditis as judged by ausculation or as estimated by exercise tolerance tests. There were no facilities for electro-cardigraphic examination. The average time away from duty for all cases was 53 days. None was seen subsequently with a relapse, or evidence of myocardial or endocardial damage.

The criticism that cardiac disease may have manifested itself later in some patients is fair, as the possibilities of adequate follow-up was for obvious reasons somewhat incomplete; but, as at least half the soldiers affected were stationed in the vicinity of the hospital for the period covered, it is equally fair to assume that, in these at least our estimation of the benignancy of their diseases was correct. The 3 cases who relapsed were as a precautionary measure downgraded for three months, as well as 5 other soldiers who persisted in complaining of residual rheumatic pain in spite of a normal B.S.R. and the absence of abnormal clinical findings. 
POSSIBLE EXCITING AND PREDISPOSING FACTORS

Eight followed acute streptococcal tonsillitis with incubation periods from 2 to 10 days. Other predisposing factors were malaria (6), dysentery (4), sandfly fever (3), but as the intervening interval before the onset of polyarthritis varied from 5 days to several weeks, the significance of these is perhaps problematical. One factor was prominent in the histories of 17 cases-severe and/or unaccustomed exposure to cold or damp, often prolonged, and these patients emphatically dated their illness from this exposure. The polyarthritis began from 2 to 14 days later. The immediate antecedent history of the remaining 9 cases failed to disclose any obvious cause. Six patients gave a history of "rheumatic fever" in childhood. One man (not included in this series) exhibited the clinical picture of rheumatic fever, except for the important difference of lack of response of the arthritic symptoms to salicylates, and was found to have a small focus of infection containing gas-forming organisms. The notes of this man are as follows:

CASE 5

Aged 24. Feb. 12: G.S.W.R. knee-operated upon. Apparently progressing favourably. There was no history of serum having been administered. Feb. 27: Onset of pains in wrist, knee, and jaw. Feb. 28: T. $102 \cdot 6^{\circ}$, P. 110 . Healthy looking wound 6 in. long inner side of L. knee and thigh. Marked effusion into both knees. Both wrists very painful on movement; complains of pain in temporo-maxillary joints on opening mouth, movements of which are limited. Sod. sal. grs. 150 daily begun. March 3: T. 101.2 ${ }^{\circ}$, P. 112. Knees as before. Marked pain in temporaro-maxillary joints. Swelling of both wrists. Pain in left shoulder and elbow. Wound explored: small abscess in adductor canal containing gas-forming organisms. March 9: T. normal, P. 90. Effusions have disappeared. Arthritic pains still present. March 20: Uninterrupted recovery; no pains.

\section{Discussion}

A primary attack of rheumatic fever is relatively uncommon in adults. Hedley (1940) found that rheumatic fever in more than $75 \%$ of cases began before the age of 20 . It is well recognized that the disease in adults does not affect the heart as frequently as in children. The 47 cases here recorded suggest that in adults, or at least in soldiers living under service conditions abroad, so-called rheumatic fever rarely causes cardiac damage. Green (1944) states: "The aetiology of rheumatic fever cannot be considered as finally solved, but it is essential that haemolytic streptococci should be placed first and foremost in all cases relating to the spread and re-activation of acute rheumatism." Most authorities agree with this. The low incidence of streptococcal infection $(17 \%)$ in my cases is in itself a cogent reason for attempting to differentiate this benign syndrome. Furthermore, the incidence of these cases bore no obvious relationship to the frequency of admissions for tonsillitis to the other wards of the hospital. The most striking possible precipitating cause was severe and prolonged exposure to cold and damp $(36 \%)$. It is suggested that this may in some way lower the body immunity so markedly that the unknown cause of acute rheumatism acts. To assume a latent virus infection is tempting, but so far no positive support has been given to this theory. Serum arthritis produces a picture resembling rheumatic fever making an allergic basis for all acute rheumatism attractive. Coburn's views on the hypersensitiveness of certain tissues to haemolytic streptococci are well known; in those of my cases, however, in which a bacterial or protozoan toxin might be suspected $(49 \%)$, the interval between the possible cause and effect is so variable that allergy or hypersensitiveness cannot be the complete answer. The points of differentiation in the 47 cases from classical rheumatic fever are: (1) relative absence of toxaemia and malaise; (2) the quick response to treatment with salicylates; (3) the rapid fall in B.S.R.; (4) the normal red blood count-in juvenile rheumatic fever a secondary anaemia is almost a constant finding; (5) the absence of cardiac complications; (6) the infrequency of relapses or previous history; (7) the relatively insignificant rôle played by the streptococci; (8) the frequency in which a history of severe exposure to severe cold and damp is obtained. The benign nature of the disease is the chief reason for thinking that this is a clinical entity and should be differentiated from the classical type. Ferguson's designation " acute febrile polyarthritis " is favoured in order to avoid confusion with the more serious true rheumatic fever.

It must be emphasized emphatically that this article deals with a special group of males, and it is not suggested that a benign type of rheumatic fever is recognizable in children. Rheumatic fever in children and adolescents should be regarded as affecting the heart until conclusively proved otherwise.

\section{Summary}

1. Forty-seven soldiers, all stationed abroad, suffering from a benign type of rheumatic fever are discussed.

2. The relative absence of malaise, the rapid fall in B.S.R., the normal red blood count, and the absence of cardiac complications, are the chief features in distinguishing the disease from classical rheumatic fever.

3. Streptococci infection was relatively infrequent as a precipitating cause; $36 \%$ gave a recent history of severe exposure to cold and damp.

4. The name acute febrile polyarthritis for this benign type of rheumatic fever is favoured.

\section{REFERENCES}

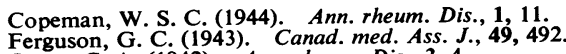
Green, C. A. (1942). Ann. rheum. Dis., 3, 4. Hedley, O. F. (1940). Publ. Hlth. Rep. Wash., 55, 1707. 